\title{
REVIEW
}

\section{The efficacy of telerehabilitation in dysphagic patients: a systematic review}

\section{L'efficacia della teleriabilitazione in pazienti con disfagia: una revisione sistematica}

\author{
S. NORDIO ${ }^{1}$, T. INNOCENTI², M. AGOSTINI' ${ }^{1}$, F. MENEGHELLO' ${ }^{1}$, I. BATTEL ${ }^{1}$ \\ ${ }^{1}$ Fondazione Ospedale San Camillo IRCCS, Venice, Italy; ${ }^{2}$ Department of Neuroscience, Rehabilitation, \\ Ophthalmology, Genetics, Maternal and Child Health, University of Genova, Italy
}

\section{SUMMARY}

Telerehabilitation is the use of telecommunications technology for rehabilitation. Recently, some studies have shown positive effects of telerehabilitation of swallowing disorders, yet there are no systematic reviews verifying the evidence. The aim of this review is to assess the effects of telerehabilitation in the field of dysphagia as an alternative to face-to-face patient care, considering swallowing recovery and/or quality of life in different patient populations. We searched the Cochrane Library, MEDLINE, EMBASE, Google Scholar, Google Search and the grey literature from inception until December 2016 for publications written in English (keywords: telerehabilitation, telemedicine, dysphagia, swallowing disorders), which resulted in 330 records. Abstract screening and data extraction was carried out independently by two reviewers. Four papers were selected to read in full, and the methodological quality of the studies included was evaluated using Cochrane Collaboration's tool for assessing risk of bias. One study met our inclusion criteria (Wall et al. 2016), which showed that telerehabilitation improves adherence to treatment compared to patient-directed intervention. Although adherence is an important factor that influences the treatment outcome, clinical outcomes have to be examined in randomised controlled trials in order to reach evidence in this field. Lastly, this systematic review did not demonstrate the efficacy of telerehabilitation compared with face-to face therapy.

KEY WORDS: Dysphagia $\bullet$ Swallowing disorders $\bullet$ Telemedicine $\bullet$ Telerehabilitation

\section{RIASSUNTO}

La teleriabilitazione consiste nell'utilizzo della tecnologia delle telecomunicazioni per la riabilitazione. Recentemente, alcuni studi hanno mostrato gli effetti positivi della teleriabilitazione sui disturbi di deglutizione, ma non esistono revisioni sistematiche che ne verifichino l'efficacia. L'obiettivo di questa revisione è quello di valutare gli effetti della teleriabilitazione nel campo della disfagia in alternativa alla cura del paziente face-to-face, rispetto al recupero del disturbo deglutitorio elo alla qualità della vita in diverse popolazioni di pazienti. Sono stati considerati i seguenti database: Cochrane Library, MEDLINE, EMBASE, Google Scholar, Google Search e la letteratura grigia fino al dicembre 2016 per pubblicazioni scritte in inglese (parole chiave: teleriabilitazione, telemedicina, disfagia, disturbi della deglutizione), che hanno rilevato 330 articoli. Lo screening degli abstract e l'estrazione dei dati sono stati eseguiti in modo indipendente da due revisori. Sono stati scelti 4 articoli per la lettura integrale; la qualità metodologica degli studi inclusi ed il risk of bias sono stati valutati utilizzando lo strumento della Cochrane "Cochrane Collaboration's tool for assessing risk of bias". Un solo studio ha soddisfatto i nostri criteri di inclusione (Wall et al. 2016), dimostrando che la teleriabilitazione migliora l'aderenza al trattamento da parte del paziente. Anche se l'aderenza rappresenta un fattore importante che influenza l'esito del trattamento stesso, i risultati dovrebbero essere esaminati in trial clinici randomizzati controllati che prendano in esame anche outcome quali il grado di severità della disfagia, al fine di ottenere prove attendibili di efficacia. In conclusione, tale revisione sistematica non dimostra l'efficacia della teleriabilitazione rispetto alla terapia face-to-face per quanto riguarda il miglioramento dei disturbi di deglutizione.

PAROLE CHIAVE: Disfagia $\bullet$ Disturbi di deglutizione $\bullet$ Telemedicina $\bullet$ Teleriabilitazione

Acta Otorhinolaryngol Ital 2018;38:79-85

\section{Introduction}

Dysphagia is a swallowing disorder that increases the risk of morbidity and mortality ${ }^{1}$. It is well documented that early intervention is crucial for preventing malnutrition and pneumo- nia $^{2}$. Nevertheless, dysphagia management is often impeded by factors such as distance from healthcare services, especially in patients who live in rural or remote areas, and timely access that could lead to an increase in dysphagia severity ${ }^{3}$. Recently, a study showed the effects on use of telerehabili- 
tation modality to facilitate dysphagia interventions, particularly in relation to the assessment ${ }^{4}$.

Telerehabilitation is the provision of rehabilitation services to patients at a remote location using information and communication technologies ${ }^{5}$. In the last decade, rapid developments in telecommunications have increased the possibility of health care delivery. Telerehabilitation involves the remote delivery of different rehabilitation services via telecommunications technology ${ }^{6}$. The benefits of telerehabilitation include the delivery of prolonged and intensive therapies tailored to patients' needs at home while at the same time making significant cost savings ${ }^{7}$. Several authors observed that the use of telerehabilitation leads to high levels of patient-reported satisfaction, reinforcing the hypothesis that the delivery of rehabilitative services at a distance is a feasible alternative to routine care ${ }^{89}$.

Despite the growing use of telerehabilitation in the field of physiotherapy and occupational therapy ${ }^{10}$, studies concerning its application in speech and language therapy are still limited. In particular, in this field the majority have focused on hearing and language disorders, and only few have addressed swallowing disorders ${ }^{11}$. Perlman and Witthawaskul ${ }^{12}$ and Malandraki et al. ${ }^{13}$ demonstrated that remote dysphagia consultations are feasible and relatively accessible from a technological standpoint. For patients with chronic and progressive neurological disorders (e.g. Parkinson's disease), periodic monitoring of communication and swallowing is important to assist them in maintaining these functions. An additional advantage of telerehabilitation in speech and language pathology is the potential to facilitate generalisation of treatment to the individual's everyday life. It is well established that early rehabilitative interventions can prevent and decrease the incidence of pneumonia in dysphagic patients ${ }^{14}$.

\section{Objectives}

The aim of this systematic review is to verify the effectiveness of telerehabilitation programmes compared to face-to-face rehabilitation treatments, in terms of swallowing recovery and/or quality of life concerning feeding.

\section{Materials and methods}

\section{Search strategy}

The review was published in PROSPERO (International Prospective register of systematic reviews) with the registration number CRD42016051662. We used the PRISMA 2009 checklist ${ }^{15}$ (Fig. 1) to establish the protocol.

We searched MEDLINE, Embase and The Cochrane Cen- tral Register of Controlled Trials (CENTRAL) from inception until December 2016 for publications written in English, using the key words: telemedicine, telerehabilitation, telemonitoring, telehealth, deglutition disorders, eating disorders, swallowing, swallowing disorders, dysphagia. Details of search terms and strategies specific for each database are available in Appendix 1.

We also searched grey literature such as Google Scholar, reference lists of articles, from inception until December 2016. We identified published and unpublished trials, by hand searching the reference lists from relevant articles.

\section{Selection criteria}

Studies were eligible for inclusion if they tested telerehabilitation for the recovery of swallowing functions in patients affected by dysphagia due to any type of impairment or disease, including randomised controlled trials (RCT) and quasi-randomised controlled trials with or without blinding of examiner(s). We excluded studies on patients with swallowing disorders due to malocclusion and atypical deglutition. We considered studies focusing on swallowing interventions using telerehabilitation, which includes therapeutic exercises, compensative manoeuvre, monitoring and counselling. In the context of this systematic review, telerehabilitation was considered as:

- provided by means of any kind of technological device;

- allowing healthcare professional/patient interaction both on-line (via a videoconferencing system, the therapist connects to the patient's device, taking control and interacting directly, giving rehabilitation session exactly as it is in the presence of the patient) or off-line (the patient performs the rehabilitation activities prescribed independently using the device);

- provided by healthcare professionals or caregivers through remote supervision.

\section{Data collection and analysis}

Two authors (SN and IB) independently screened the titles and abstracts of the 330 records retrieved from the search strategy, applying the selection criteria previously described. The full text of the possible eligible records were retrieved and analysed for final inclusion in this systematic review. Any disagreement was resolved through discussion and contacting a third author (TI), if needed. Two authors (SN and IB) independently extracted the data from the included study, using a standard form, in which the items extracted were: details of the participants (i.e. age, gender, type of disease); inclusion/exclusion criteria for patients' eligibility; duration, intensity and frequency of interventions and controls; description of telerehabilitation programme; outcomes assessed. 


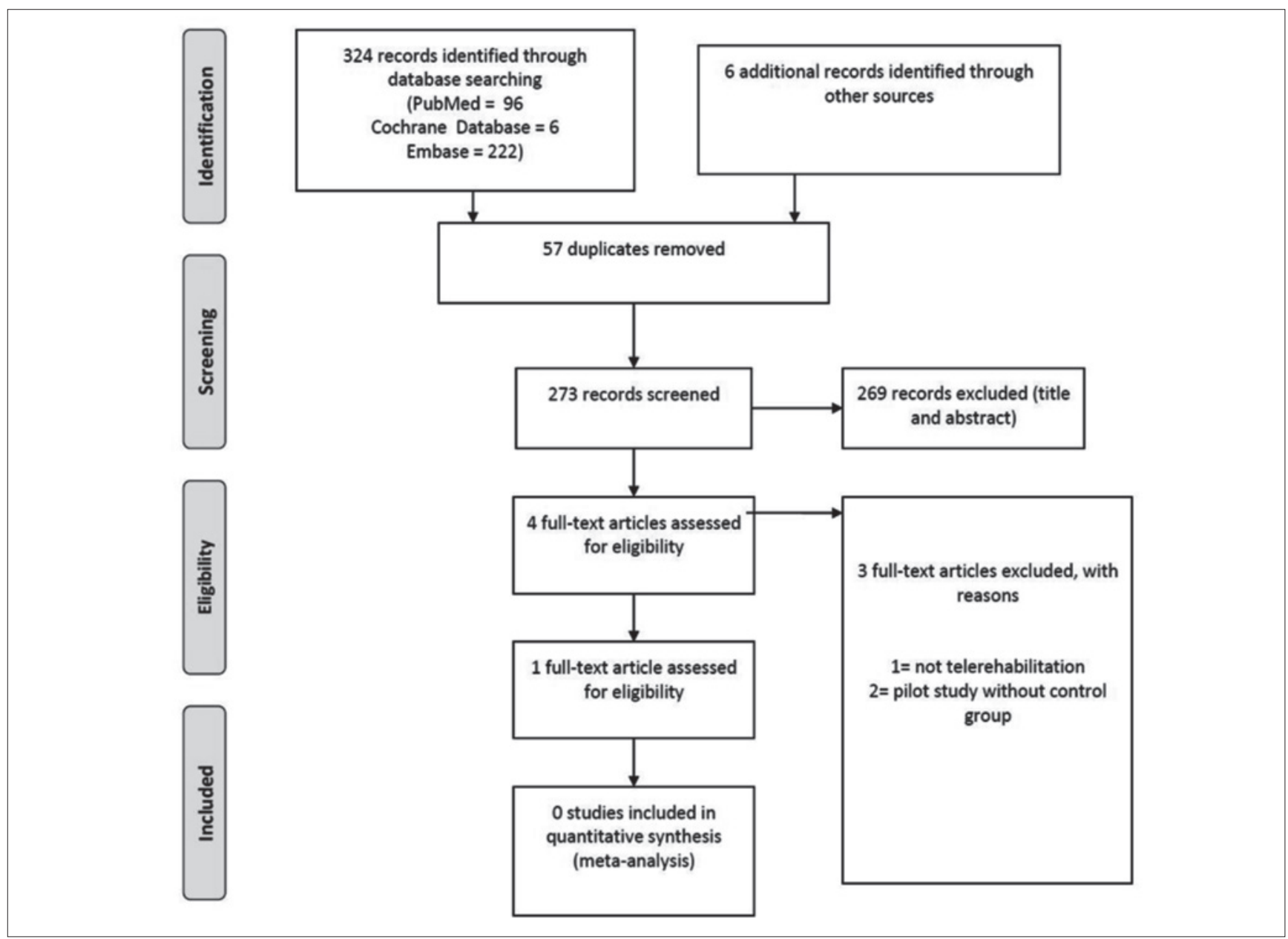

Fig. 1. Prisma flow chart.

\section{Quality assessment}

Two authors (SN and IB) independently evaluated the methodological quality of the included studies, using the Cochrane Collaboration's tool for assessing risk of bias ${ }^{16}$. Quality assessment of studies was focused on areas of bias which might overestimate the effectiveness of interventions. The domains considered as relevant: random sequence generation; allocation concealment; blinding of participants and personnel; blinding of outcome assessment; incomplete outcome data, selective reporting, other sources of bias.

\section{Results}

\section{Selection of studies}

The literature search retrieved 330 records (i.e. MEDLINE $=96$; embase $=222$; CENTRAL $=6$; grey literature $=6$ ). After the removal of 57 duplicates, we screened the title and abstract of 273 references and selected 4 pa- pers, for which we assessed the full text for final inclusion. Among these, 3 papers were excluded for the following reasons. Two studies did not have a control group ${ }^{17} 18$. One study was excluded because it was not a proper telerehabilitation intervention ${ }^{19}$; the authors conducted videoassisted swallowing therapy, but there was not a remote device for telerehabilitation.

Finally, only one RCT ${ }^{20}$ presented the criteria for inclusion in this review (Fig. 1). Hence, it was not possible to carry out a meta-analysis, and we so carried out only a descriptive analysis of the study selected.

\section{Characteristics of the included study}

The main characteristics of the RCT included are described in Table I. Wall et al.'s ${ }^{20}$ study included 79 patients with oropharyngeal squamous cell carcinoma, comparing 3 groups and examining adherence to a prophylactic swallowing therapy protocol ("Pharyngocise" therapy protocol 21 , across three different service delivery models: clini- 
Table I. Characteristics of the studies included.

\begin{tabular}{|c|c|c|c|c|c|}
\hline $\begin{array}{l}\text { Author } \\
\text { Year } \\
\text { Design }\end{array}$ & $\begin{array}{l}\text { No. and characteristics } \\
\text { of participants }\end{array}$ & $\begin{array}{l}\text { Groups and types } \\
\text { of interventions }\end{array}$ & Endpoint(s) & $\begin{array}{l}\text { Assessments } \\
\text { and follow-up }\end{array}$ & Results \\
\hline $\begin{array}{l}\text { Wall LR et al. } 2016 \\
\text { RCT }\end{array}$ & $\begin{array}{l}79 \text { adults } \\
8 \text { drop out } \\
63 \mathrm{M}, 8 \mathrm{~F} \\
\mathrm{M}=56.92 \text { years } \\
\text { Inclusion criteria: } \\
\text { adult patients diagnosed } \\
\text { with oropharyngeal } \\
\text { squamous cell carcinoma } \\
\text { and planned for non- } \\
\text { surgical curative intent. } \\
\\
\text { Exclusion criteria: } \\
\text { severe cognitive deficits, } \\
\text { non-English speaking, } \\
\text { significant vision, hearing } \\
\text { or physical dexterity } \\
\text { impairments deemed by } \\
\text { the radiation oncology } \\
\text { physician at a level that } \\
\text { prohibited participation }\end{array}$ & $\begin{array}{l}3 \text { subgroups of } 26 \text { patients } \\
\text { attending "pharyngocise" } \\
\text { therapy protocol: } \\
1 \text { clinician-directed face- } \\
\text { to-face therapy; } \\
2 \text { technology assisted } \\
\text { therapy model } \\
\text { using a purpose- } \\
\text { built asynchronous } \\
\text { telepractice application, } \\
\text { Swallow-it; } \\
3 \text { independent patient self- } \\
\text { directed therapy. } \\
\text { Duration: } 5 \text { days } \\
\text { for } 6 \text { weeks. } \\
\text { Total: } 240 \text { cycles }\end{array}$ & $\begin{array}{l}\text { Adherence to } \\
\text { prophylactic } \\
\text { swallowing } \\
\text { exercise } \\
\text { protocol. } \\
\text { It was } \\
\text { measured } \\
\text { differently } \\
\text { depending on } \\
\text { the treatment: } \\
\text { - daily in } \\
\text { purpose- } \\
\text { built } \\
\text { exercise log } \\
\text { books; } \\
\text { - numbers of } \\
\text { completed } \\
\text { cycles } \\
\text { into the } \\
\text { Swallow-it } \\
\text { system }\end{array}$ & $\begin{array}{l}\text { Adherence was } \\
\text { measured every week } \\
\text { for six weeks }\end{array}$ & $\begin{array}{l}\text { Significant decline in } \\
\text { adherence over time } \\
(p<0.001) \text { in all groups, } \\
\text { without significant } \\
\text { differences among groups. } \\
\text { For weeks 1-3: } \\
1 \text { clinician-directed versus } \\
\text { Swallow-it does not } \\
\text { show any significant } \\
\text { difference ( } p=0.466) ; \\
2 \text { clinician-directed model } \\
\text { showed significantly } \\
\text { better adherence than } \\
\text { patient self-directed } \\
\text { therapy ( } p=0.014) ; \\
3 \text { Swallow-it presented } \\
\text { a trend of higher } \\
\text { adherence than } \\
\text { patients-direct, but not } \\
\text { significant difference } \\
\text { ( } p=0.064) \text {. } \\
\text { For weeks 4-6: } \\
\text { there was no significant } \\
\text { difference between the } \\
\text { groups ( } p=0.391 \text { ) }\end{array}$ \\
\hline
\end{tabular}

cian-directed face-to-face therapy, a technology-assisted therapy model using a telepractice application (Swallowit), and at least an independent patient-directed therapy. The duration of the therapy was five sessions for six weeks. Adherence was measured at the end of every week.

\section{Risk of bias assessment}

Figure 2 summarises the assessment of the methodological quality of the study, which is an RCT. There is no risk of selection bias due to an adequate random sequence generation and allocation of the randomisation sequence. We found performance and detection bias due to absence of blindness, and attrition bias due to unbalanced dropouts between the groups. In addition, intention to treat analysis was not applied. The risk of reporting bias is unclear because the study protocol is not available and there is insufficient information to permit judgement of partition of primary and secondary outcomes.

\section{Effects of interventions}

The authors found a significant decline in adherence over time $(\mathrm{p}<0.001)$ in all groups, and no significant difference in the overall percent of exercise completed by the service-delivery group $(\mathrm{p}=0.107)$. Regarding adherence for weeks 1-3, the results are as follows: the clinician-directed model showed significantly greater adherence than patient self-directed therapy $(\mathrm{p}=0.014)$, while cliniciandirected versus Swallow-it did not show any significant difference $(\mathrm{p}=0.466)$. Finally, Swallow-it presented a trend towards higher adherence than patient-directed, but the difference was not significant $(p=0.064)$. For weeks 4-6, they found adherence was not significantly different between groups $(\mathrm{p}=0.391)$.

\section{Discussion}

Despite the emerging number of telerehabilitation studies on dysphagic patients ${ }^{17-19}$, we found only one study that was eligible for inclusion in this systematic review ${ }^{20}$. The aims of the included study were to verify the adherence to a prophylactic swallowing exercise protocol and if clinical and demographic factors influence adherence. Thus, this study did not report any swallowing outcomes and/or swallowing improvements, which are essential to verify the effectiveness of a treatment. They found that clinician-directed treatment obtained the highest adherence compared with telerehabilitation (Swallow-it) and 
Random sequence generation (selection bias)

Allocation concealment (selection bias)

Blinding of participants and personnel (performance bias)

Blinding of outcome assessment (detection bias)

Incomplete outcome data (attrition bias)

Selective reporting (reporting bias)

Other bias

Fig. 2. Risk of bias assessment (from Wall et al., $2016^{20}$, mod.).

patient-direct therapy. Nevertheless, there was a trend for greater adherence in telerehabilitation than patientdirect. This finding suggests that patients may be more motivated and encouraged when they have access to a telepractice service model instead of independent therapy. Although adherence represents an important value for positive treatment outcomes, this study had some methodological limitations. First, the criteria for adherence assessment varied depending on the treatments, resulting in difficulties comparing outcomes. Moreover, in the patient-directed group, adherence was calculated subjectively through the number of exercises reported by each patient in log books, thus increasing the risk of bias. The multivariable analysis was based only on variables taken prior to chemotherapy, and so other variables could have influenced adherence. In addition, during data collection, there were some dropouts that resulted in heterogeneous sample sizes.

The results of our systematic review suggest that telerehabilitation in the field of dysphagia treatment is at an initial phase. We considered it important to highlight the value of this topic in the field of deglutition disorders, with objective data from a systematic review, because of the relevance of technological tools for telerehabilitation that could be implemented in future studies.

Several limitations of this review should be acknowledged. First of all, most of the studies in telerehabilitation concerning dysphagia are characterised by small sample sizes, large variability of illnesses included, heterogeneity of treatments and outcomes and the consistent presence of biases. The limitation of small samples is common in the rehabilitation literature concerning dysphagia, because of the different diagnoses that can cause it, the large range of severity and disability experienced by patients and the long time needed to observe changes in clinical outcomes ${ }^{7}$. In addition, we chose to distinguish telerehabilitation from other telemedicine applications (e.g. telemonitoring, teleradiology) because of the possibility of providing therapeutic interventions, remotely controlled by healthcare professionals, with rehabilitation intent. Moreover, we only considered trials reported in English, which restricted the raw dataset of records used for screening.

\section{Conclusions}

The systematic review did not provide final evidence on the efficacy of telerehabilitation in dysphagia recovery. Undoubtedly, in the last years telerehabilitation is becoming an interesting model of care even in speech and language therapy, which has been examined by several studies ${ }^{311}$. Specifically, telemedicine offers an innovative approach to increase access to clinical rehabilitation medicine services and to guarantee adequate services to people with swallowing disorders, which require periods of intensive treatment followed by long-term management. Further, it is considered more motivating to receive rehabilitation in a patient's own environment ${ }^{8}$. For these reasons, though face-to-face intervention has always been considered the 'gold standard' of care, it is necessary to consider alternative types of service delivery as supplement or replacement of face-to-face treatments in some cases. Considering telerehabilitation in other fields, encouraging data can be seen, and the only one RCT ${ }^{20}$ paper we found by the systematic review suggests good compliance of patients who attended telerehabilitation. Notwithstanding, additional research in the field of dysphagia is needed to define the benefits and risks associated with assisting patients in telerehabilitation modality. Future trials need to have a more robust design and evaluate the effectiveness of treatments based on specific clinical outcomes and quality of life, in different populations of patients with different type and severity of dysphagia, and associated with cost accountability and cost effectiveness analysis.

\section{Acknowledgements}

We would like to thank Dr. Katie Palmer for the support in this study.

\section{References}

1 Gonzalez-Fernandez M, Daniels SK. Dysphagia in stroke and neurologic disease. Phys Med Rehabil Clin N Am 2008; 19:867-88.

2 Bours GJ, Speyer R, Lemmens J, et al. Bedside screening tests vs videofluoroscopy or fibreoptic endoscopic evaluation of 
swallowing to detect dysphagia in patients with neurological disorders: systematic review. J Adv Nurs 2009;65:477-93.

3 Mashima PA, Brown JE. Remote management of voice and swallowing disorders. Otolaryngol Clin North Am 2011;44:1305-16.

4 Ward EC, Burns CL, Theodoros DG, et al. Impact of dysphagia severity on clinical decision making via telerehabilitation. Telemed J E Health 2014;20:296-303.

5 Brennan DM, Mawson S, Brownsell S. Telerehabilitation: enabling the remote delivery of healthcare, rehabilitation, and self management. Stud Health Technol Inform 2009;145:231-48.

6 Rogante M, Grigioni M, Cordella D, et al. Ten years of telerehabilitation: a literature overview of technologies and clinical applications. Neuro Rehabil 2010;27:287-304.

7 Dansky KH, Palmer L, Shea D, et al. Cost analysis of telehomecare. Telemed J E Health 2001;7:225-32.

8 Piron L, Turolla A, Tonin P, et al. Satisfaction with care in post-stroke patients undergoing a telerehabilitation programme at home. J Telemed Telecare 2008;14:257-60.

9 Laver KE, Schoene D, Crotty M, et al. Telerehabilitation services for stroke. Cochrane Database Syst Rev 2013:12:CD010255.

10 Agostini M, Moja L, Banzi R, et al. Telerehabilitation and recovery of motor function: a systematic review and metaanalysis. J Telemed Telecare 2015;21:202-13.

11 Molini-Avejonas DR, Rondon-Melo S, Amato CA, et al. A systematic review of the use of telehealth in speech, language and hearing sciences. $\mathrm{J}$ Telemed Telecare 2015;21:367-76.

12 Perlman AL, Witthawaskul W. Real-time remote telefluoroscopic assessment of patients with dysphagia. Dysphagia 2002;17:162-7.

13 Malandraki GA, McCullough G, He X, et al. Teledynamic evaluation of oropharyngeal swallowing. J Speech Lang Hear Res 2011;54:1497-505.

14 Daniels SK, Pathak S, Rosenbek JC, et al. Rapid aspiration screening for suspected stroke. Part 1: Development and Validation. Arch Phys Med Rehabil 2016;97:1440-8.

15 Moher D, Shamseer L, Clarke M, et al. Preferred reporting items for systematic review and meta-analysis protocols (PRISMA-P) 2015 statement. Syst Rev 2015;4:1.

16 Higgins JP, Altman DG, Gotzsche PC, et al. The Cochrane Collaboration's tool for assessing risk of bias in randomised trials. BMJ 2011;343:d5928.

17 Wall LR, Ward EC, Cartmill B, et al. Examining user perceptions of SwallowIT: a pilot study of a new telepractice application for delivering intensive swallowing therapy to head and neck cancer patients. J Telemed Telecare 2017;23:53-9.

18 Clawson B, Selden M, Lacks M, et al. Complex pediatric feeding disorders: using teleconferencing technology to improve access to a treatment program. Pediatr Nurs 2008;34:213-6.

19 Manor Y, Mootanah R, Freud D, et al. Video-assisted swallowing therapy for patients with Parkinson's disease. Parkinsonism Relat Disord 2013;19:207-11.

20 Wall LR, Ward EC, Cartmill B, et al. Adherence to a prophylactic swallowing therapy program during (chemo) radiotherapy: impact of service-delivery model and patient factors. Dysphagia 2016;32:279-92.

21 Carnaby-Mann G, Crary MA, Schmalfuss I, et al. "Pharyngocise": randomized controlled trial of preventative exercises to maintain muscle structure and swallowing function during head-and-neck chemoradiotherapy. Int J Radiat Oncol Biol Phys 2012;83:210-9. 
Appendix 1. Electronic searches

MEDLINE

Telemedicine (Mesh) OR Telerehabilitation (Mesh) OR Telerehabilitation OR Telemedicine OR Telemonitoring OR Telehealth AND Deglutition Disorders (Mesh) OR Deglutition disorders OR Eating disorders OR Swallowing OR Swallowing disorders OR Dysphagia

EMBASE

Telemedicine OR Telerehabilitation OR Telemonitoring OR Telehealth AND Deglutition disorders OR Eating disorders OR swallowing OR Swallowing disorders OR Dysphagia

THE COCHRANE LIBRARY

Results of the free words research were collected together:

- dysphagia telerehabilitation;

- eating disorders telerehabilitation;

- deglutition disorders and swallowing disorders telerehabilitation;

- eating disorders telemedicine;

- swallowing disorders and deglutition disorders telemedicine;

- dysphagia disorders telemedicine. 